\title{
Complementarity of the Maldacena and Randall-Sundrum pictures
}

\author{
M J Duff and James T Liu \\ Randall Laboratory, Department of Physics, University of Michigan, Ann Arbor, MI 48109-1120, \\ USA \\ E-mail: mduff@umich.edu and jimliu@umich.edu
}

Received 22 January 2001

Published 1 August 2001

Online at stacks.iop.org/CQG/18/3207

\begin{abstract}
We revive an old result, that one-loop corrections to the graviton propagator induce $1 / r^{3}$ corrections to the Newtonian gravitational potential, and compute the coefficient due to closed loops of the $U(N) \mathcal{N}=4$ super-Yang-Mills theory that arises in Maldacena's AdS/CFT correspondence. We find exact agreement with the coefficient appearing in the Randall-Sundrum brane-world proposal. This provides more evidence for the complementarity of the two pictures.
\end{abstract}

PACS numbers: $1110 \mathrm{~K}, 1110 \mathrm{G}, 1125 \mathrm{M}$

It is an old, and seemingly forgotten result that one-loop corrections to the graviton propagator induce $1 / r^{3}$ corrections to the gravitational potential $[1,2]$ :

$$
V(r)=\frac{G m_{1} m_{2}}{r}\left(1+\frac{\alpha G}{r^{2}}\right),
$$

where $G$ is the four-dimensional Newton constant, $\hbar=c=1$ and $\alpha$ is a purely numerical coefficient given, in the case of spins $s \leqslant 1$, by $45 \pi \alpha=12 N_{1}+3 N_{1 / 2}+N_{0}$, where $N_{s}$ are the numbers of particle species of spin $s$ going around the loop [3-5]. However, the importance of this result has recently become apparent in attempts [6-10] to relate two topical but, at first sight, different developments in quantum gravity. These are Maldacena's AdS/CFT correspondence [11-13] and the Randall-Sundrum brane-world mechanism [14].

The AdS/CFT correspondence in general relates the gravitational dynamics of a $(d+1)$ dimensional anti-de Sitter spacetime, $\mathrm{AdS}_{d+1}$, to a $d$-dimensional conformal field theory, $\mathrm{CFT}_{d}$. In the case of $d=4$, Maldacena's conjecture, based on the decoupling limit of D3-branes in type IIB string theory compactified on $S^{5}$, then relates the dynamics of $\mathrm{AdS}_{5}$ to an $\mathcal{N}=4$ superconformal $U(N)$ Yang-Mills theory on its four-dimensional boundary [11]. 
Other compactifications are also possible, leading to different SCFTs on the boundary. We note that, by choosing Poincaré coordinates on $\mathrm{AdS}_{5}$, the metric may be written as

$$
\mathrm{d} s^{2}=\mathrm{e}^{-2 y / L}\left(\mathrm{~d} x^{\mu}\right)^{2}+\mathrm{d} y^{2},
$$

in which case the superconformal Yang-Mills theory is taken to reside at the boundary $y \rightarrow-\infty$.

The Randall-Sundrum mechanism, on the other hand, was originally motivated, not via the decoupling of gravity from D3-branes, but rather as a possible mechanism for evading Kaluza-Klein compactification by localizing gravity in the presence of an uncompactified extra dimension. This was accomplished by inserting a positive tension 3-brane (representing our spacetime) into $\mathrm{AdS}_{5}$. In terms of the Poincaré patch of $\mathrm{AdS}_{5}$ given above, this corresponds to removing the region $y<0$, and either joining on a second partial copy of $\mathrm{AdS}_{5}$, or leaving the brane at the end of a single patch of $\mathrm{AdS}_{5}$. In either case the resulting Randall-Sundrum metric is given by

$$
\mathrm{d} s^{2}=\mathrm{e}^{-2|y| / L}\left(\mathrm{~d} x^{\mu}\right)^{2}+\mathrm{d} y^{2},
$$

where $y \in(-\infty, \infty)$ or $y \in[0, \infty)$ for a 'two-sided' or 'one-sided' Randall-Sundrum brane, respectively.

The similarity of these two scenarios led to the notion that they are, in fact, closely tied together. To make this connection clear, consider the one-sided Randall-Sundrum brane. By introducing a boundary in $\mathrm{AdS}_{5}$ at $y=0$, this model is conjectured to be dual to a cut-off CFT coupled to gravity, with $y=0$, the location of the Randall-Sundrum brane, providing the ultraviolet cut-off. This extended version of the Maldacena conjecture [15] then reduces to the standard AdS/CFT duality as the boundary is pushed off to $y \rightarrow-\infty$, whereupon the cut-off is removed and gravity becomes completely decoupled. Note, in particular, that this connection involves a single CFT at the boundary of a single patch of $\mathrm{AdS}_{5}$. For the case of a brane sitting between two patches of $\mathrm{AdS}_{5}$, one would instead require two copies of the CFT, one for each of the patches.

It has been suggested [7-9] that a crucial test of this Randall-Sundrum version of the Maldacena conjecture would be to compare the $1 / r^{3}$ corrections to Newton's law in both pictures. From the above, we see that the contribution of a single CFT, with $\left(N_{1}, N_{1 / 2}, N_{0}\right)=$ $\left(N^{2}, 4 N^{2}, 6 N^{2}\right)$, is

$$
V(r)=\frac{G m_{1} m_{2}}{r}\left(1+\frac{2 N^{2} G}{3 \pi r^{2}}\right) .
$$

Using the AdS/CFT relation $N^{2}=\pi L^{3} / 2 G_{5}$ [11] and the one-sided brane-world relation $G=2 G_{5} / L[8,14]$, where $G_{5}$ is the five-dimensional Newton's constant and $L$ is the radius of $\mathrm{AdS}_{5}$, this becomes

$$
V(r)=\frac{G m_{1} m_{2}}{r}\left(1+\frac{2 L^{2}}{3 r^{2}}\right) .
$$

The coefficient of the $1 / r^{3}$ term is $\frac{2}{3}$ of the Randall-Sundrum result quoted in [14], but, in fact, agrees with the more thorough analysis of [16]. We shall confirm below that a more careful analysis of the Randall-Sundrum picture using the results of $[9,17]$ yields exactly the same answer as the above AdS/CFT calculation, thus providing strong evidence for the conjectured duality of the two pictures.

First we derive (4) in more detail by computing the lowest-order quantum corrections to solutions of Einstein's equations. Working with linearized gravity, we begin by writing the metric as

$$
g_{\mu \nu}=\eta_{\mu \nu}+h_{\mu \nu}
$$


so that

$$
\sqrt{-g} g^{\mu v} \equiv \tilde{g}^{\mu v}=\eta^{\mu v}-\tilde{h}^{\mu v}+\cdots
$$

where

$$
\tilde{h}_{\mu \nu}=h_{\mu \nu}-\frac{1}{2} \eta_{\mu \nu} h_{\alpha}^{\alpha}
$$

In harmonic gauge, $\partial_{\mu} \tilde{g}^{\mu \nu}=0$ (i.e. $\partial_{\mu} \tilde{h}^{\mu \nu}=0$ ), the classical linearized Einstein equation reads

$$
\square \tilde{h}_{\mu \nu}^{c}(x)=-16 \pi G T_{\mu \nu}(x),
$$

where the superscript $c$ denotes the classical contribution. Fourier transforming to momentum space results in

$$
\tilde{h}_{\mu \nu}^{c}(p)=-16 \pi G \Delta_{4}(p) T_{\mu \nu}(p),
$$

where $\Delta_{4}(p)=-1 / p^{2}$ is the four-dimensional massless scalar propagator.

Incorporating one-loop corrections, the quantum-corrected metric becomes

$$
\tilde{h}_{\mu \nu}=\tilde{h}_{\mu \nu}^{c}+\tilde{h}_{\mu \nu}^{q}
$$

where the quantum correction $\tilde{h}_{q}^{\mu \nu}$ is given in momentum space by

$$
\tilde{h}_{q}^{\mu \nu}(p)=D^{\mu \nu \alpha \beta}(p) \Pi_{\alpha \beta \gamma \delta}(p) \tilde{h}_{c}^{\gamma \delta}(p) .
$$

$D^{\mu \nu \alpha \beta}$ is the graviton propagator,

$$
D^{\mu \nu \alpha \beta}(p)=\frac{1}{2} \Delta_{4}(p)\left(\eta^{\mu \alpha} \eta^{\nu \beta}+\eta^{\mu \beta} \eta^{\nu \alpha}-\eta^{\mu \nu} \eta^{\alpha \beta}+\cdots\right),
$$

and $\Pi_{\alpha \beta \gamma \delta}$ is the one-loop graviton self-energy, which by symmetry and Lorentz invariance must be of the general form

$$
\begin{array}{r}
\Pi_{\alpha \beta \gamma \delta}(p)=p^{4}\left[\Pi_{1}\left(p^{2}\right) \eta_{\alpha \beta} \eta_{\gamma \delta}+\Pi_{2}\left(p^{2}\right)\left(\eta_{\alpha \gamma} \eta_{\beta \delta}+\eta_{\alpha \delta} \eta_{\beta \gamma}\right)+\Pi_{3}\left(p^{2}\right)\left(\eta_{\alpha \beta} \hat{p}_{\gamma} \hat{p}_{\delta}+\eta_{\gamma \delta} \hat{p}_{\alpha} \hat{p}_{\beta}\right)\right. \\
\left.+\Pi_{4}\left(p^{2}\right)\left(\eta_{\alpha \gamma} \hat{p}_{\beta} \hat{p}_{\delta}+\eta_{\alpha \delta} \hat{p}_{\beta} \hat{p}_{\gamma}+\eta_{\beta \gamma} \hat{p}_{\alpha} \hat{p}_{\delta}+\eta_{\beta \delta} \hat{p}_{\alpha} \hat{p}_{\gamma}\right)+\Pi_{5}\left(p^{2}\right) \hat{p}_{\alpha} \hat{p}_{\beta} \hat{p}_{\gamma} \hat{p}_{\delta}\right] .
\end{array}
$$

The ellipses in (13) refer to gauge-dependent terms in the propagator which make no contribution if coupled to conserved sources. Combining (12)-(14), one thus obtains the quantum-corrected metric in the form

$h_{\mu \nu}^{q}(p)=-p^{2}\left[2 \Pi_{2}(p) \delta_{\mu}^{\alpha} \delta_{\nu}^{\beta}+\Pi_{1}(p) \eta_{\mu \nu} \eta^{\alpha \beta}+\left(\Pi_{3}(p)+\cdots\right) \hat{p}_{\mu} \hat{p}_{\nu} \eta^{\alpha \beta}\right] \tilde{h}_{\alpha \beta}^{c}$,

where non-physical gauge-dependent terms have again been dropped. Finally, combining both classical and one-loop quantum results at the linearized level yields

$$
\begin{aligned}
& h_{\mu \nu}(p)=-16 \pi G \Delta_{4}(p)\left[T_{\mu \nu}(p)-\frac{1}{2} \eta_{\mu \nu} T_{\alpha}^{\alpha}(p)\right] \\
& -16 \pi G\left[2 \Pi_{2}(p) T_{\mu \nu}(p)+\Pi_{1}(p) \eta_{\mu \nu} T_{\alpha}^{\alpha}(p)\right] .
\end{aligned}
$$

Note that we have ignored the gauge-dependent term in $h_{\mu \nu}$ proportional to $\hat{p}_{\mu} \hat{p}_{\nu}$. It makes no contribution when $h_{\mu \nu}$ is attached to a conserved source $T_{\mu \nu}$ satisfying $p^{\mu} T_{\mu \nu}=p^{\nu} T_{\mu \nu}=$ 0 .

The actual form of the one-loop $\Pi_{i} \mathrm{~s}$ depend on the theory at hand. However, for any massless theory in four dimensions, after cancelling the infinities with the appropriate counterterms, the finite remainder must necessarily have the form

$$
\Pi_{i}(p)=32 \pi G\left(a_{i} \ln \frac{p^{2}}{\mu^{2}}+b_{i}\right),
$$


where $a_{i}$ and $b_{i}(i=1,2,3,4,5)$, are numerical coefficients and $\mu$ is an arbitrary subtraction constant having the dimensions of mass. In order to make connection with the Newtonian potential, we Fourier transform (16) back to coordinate space. For the static potential we obtain the expected $1 / r$ behaviour at the classical level, while the quantum term generates the claimed $1 / r^{3}$ correction. In addition, the constant parts in (17) give rise to a regulator-dependent $\delta^{3}(\boldsymbol{r})$ contact interaction. However, we have no real expectation that this one-loop perturbative result remains valid when continued down to zero size. Moreover, possible $r^{-3} \ln \mu r$ terms come only from the $\hat{p}_{\mu} \hat{p}_{v}$ terms in (15) and hence drop out. For a point source, $T_{00}(x)=m \delta^{3}(r)$, we obtain to this order

$$
\begin{aligned}
& g_{00}=-\left(1-\frac{2 G m}{r}-\frac{2 \alpha G^{2} m}{r^{3}}\right), \\
& g_{i j}=\left(1+\frac{2 G m}{r}+\frac{2 \beta G^{2} m}{r^{3}}\right) \delta_{i j},
\end{aligned}
$$

where, in agreement with [2], $\alpha=4 \cdot 32 \pi\left(a_{1}+2 a_{2}\right)$ and $\beta=-4 \cdot 32 \pi a_{1}$. This yields the potential given in (1). Explicit calculations of the self-energy (17) for spin 1 [3], spin $\frac{1}{2}$ (two-component fermions) [4] and (real conformally coupled) spin 0 [5] yield ${ }^{1}$

$$
\begin{aligned}
a_{i}(s=1) & =4 a_{i}\left(s=\frac{1}{2}\right)=12 a_{i}(s=0) \\
& =\frac{1}{120(4 \pi)^{2}}(-2,3,2,-3,4) .
\end{aligned}
$$

Note that all spins contribute with the same sign as they must by general positivity arguments on the self-energy [4]. Thus

$$
\alpha=2 \beta=\frac{1}{45 \pi}\left(12 N_{1}+3 N_{1 / 2}+N_{0}\right)=\frac{2 N^{2}}{3 \pi},
$$

as quoted in the introductory paragraph above.

This $\alpha$ coefficient also determines that part of the Weyl anomaly [18, 19] involving the square of the Weyl tensor [20,21]:

$$
g_{\mu \nu}\left\langle T^{\mu \nu}\right\rangle=b\left(F+\frac{2}{3} \square R\right)+b^{\prime} G,
$$

where

$$
\begin{aligned}
& F=C_{\mu \nu \rho \sigma} C^{\mu \nu \rho \sigma}=R_{\mu \nu \rho \sigma} R^{\mu \nu \rho \sigma}-2 R_{\mu \nu} R^{\mu \nu}+R^{2}, \\
& G=* R_{\mu \nu \rho \sigma} * R^{\mu \nu \rho \sigma}=R_{\mu \nu \rho \sigma} R^{\mu \nu \rho \sigma}-4 R_{\mu \nu} R^{\mu \nu}+\frac{1}{3} R^{2},
\end{aligned}
$$

and where $b$ and $b^{\prime}$ are constants

$$
\begin{aligned}
& b=\frac{1}{120(4 \pi)^{2}}\left[12 N_{1}+3 N_{1 / 2}+N_{0}\right], \\
& b^{\prime}=-\frac{1}{720(4 \pi)^{2}}\left[124 N_{1}+11 N_{1 / 2}+2 N_{0}\right] .
\end{aligned}
$$

Note that for the $\mathcal{N}=4$ SCFT, the coefficient of the (Riemann) ${ }^{2}$ term, $b+b^{\prime}$, vanishes [21]. The same result is obtained if one calculates the holographic Weyl anomaly using the AdS/CFT correspondence [22]. Thus $b=3 \alpha / 128 \pi=c /(4 \pi)^{2}$, where the $c$ is the central charge

1 Note that a symmetry factor of $\frac{1}{2}$ was omitted in [3]; this was subsequently corrected in [5]. 
given in the normalization of [8]. For the central charge, one obtains $c=\pi L^{3} / 8 G_{5}$ [22], so that

$$
G \alpha=\frac{G L^{3}}{3 G_{5}}=\frac{2 L^{2}}{3},
$$

where the second equality makes use of the brane-world relation $G=2 G_{5} / L$. Although we have focused on the $\mathcal{N}=4$ SCFT to relate the coefficient appearing in Newton's law to the central charge, the result (24) is universal, being independent of which particular CFT appears in the AdS/CFT correspondence, which is just as well since the Randall-Sundrum coefficient does not depend on the details of the fields propagating on the brane.

We now turn to this brane-world, where the five-dimensional action has the form [14]

$$
S=\int \mathrm{d}^{5} x \sqrt{-g_{(5)}}\left[M^{3} R_{(5)}-\Lambda\right]+\int \mathrm{d}^{4} x \sqrt{-g_{(4)}} \mathcal{L}_{\text {brane }} .
$$

Here $M$ is the five-dimensional Planck mass, $M^{3}=1 /\left(16 \pi G_{5}\right)$, and $\Lambda$ is the cosmological constant in the bulk. Small fluctuations of the metric on the brane may be represented by $[9,14]$

$$
\mathrm{d} s^{2}=\mathrm{e}^{-2|y| / L}\left[\eta_{\mu \nu}+h_{\mu \nu}(x, y)\right] \mathrm{d} x^{\mu} \mathrm{d} x^{\nu}+\mathrm{d} y^{2},
$$

where $L$ is the 'radius' of AdS,

$$
R_{M N P Q}^{(5)}=-\frac{1}{L^{2}}\left(g_{M P}^{(5)} g_{N Q}^{(5)}-g_{M Q}^{(5)} g_{N P}^{(5)}\right),
$$

and is related to $\Lambda$ by $\Lambda=-12 M^{3} / L^{2}$. The brane-world geometry has been chosen such that $x^{\mu}$ are coordinates along the 3-brane, while $y$ is the coordinate perpendicular to the brane (which sits at $y=0$ ).

Both brane and bulk quantities are contained in the linearized metric $h_{\mu \nu}(x, y)$. However, for comparison with the CFT on the brane, we are only concerned with the former. Hence we consider a matter source on the brane, and examine $h_{\mu \nu}(x) \equiv h_{\mu \nu}(x, y=0)$. For this case, the results of $[9,17]$ indicate

$h_{\mu \nu}(p)=-\frac{2}{L M^{3}} \Delta_{4}(p)\left[T_{\mu \nu}(p)-\frac{1}{2} \eta_{\mu \nu} T_{\alpha}^{\alpha}(p)\right]-\frac{1}{M^{3}} \Delta_{K K}(p)\left[T_{\mu \nu}(p)-\frac{1}{3} \eta_{\mu \nu} T_{\alpha}^{\alpha}(p)\right]$.

This expression has a clear physical meaning; $\Delta_{4}(p)$, the four-dimensional massless propagator, corresponds to the zero-mode graviton localized on the brane, while

$$
\Delta_{K K}(p)=-\frac{1}{p} \frac{K_{0}(p L)}{K_{1}(p L)}
$$

is the propagator for the continuum Kaluza-Klein graviton modes. Comparing the first term of (28) to (16), we obtain the relation between four- and five-dimensional Newton's constants, $G=2 G_{5} / L=1 /\left(8 \pi L M^{3}\right)$ given above. Note that in the above we have taken the brane to be at the end of a single patch of $\mathrm{AdS}_{5}$, as was done in $[8,9]$. This corresponds to the case at hand, since the AdS/CFT relations we have employed above pertain to a single copy of $\mathrm{AdS}_{5}$.

The continuum graviton modes give rise to corrections to the Newtonian potential. At large distances, corresponding to $p L \ll 1$, a small argument expansion for Bessel functions yields

$$
\Delta_{K K}(p)=\frac{L}{2}\left(\ln \frac{p^{2} L^{2}}{4}+2 \gamma\right)+\mathcal{O}\left(p^{2}\right)
$$


and, just as in (17), is the source of the $1 / r^{3}$ correction to the Newtonian potential. For a static gravitational source of mass $m$ on the brane, $T_{00}(p)=2 \pi \delta\left(p_{0}\right) m$, evaluating the Fourier transform for $r \gg L$ yields the linearized metric [16]

$$
\begin{aligned}
& h_{00}=\frac{2 G m}{r}\left(1+\frac{2 L^{2}}{3 r^{2}}+\cdots\right), \\
& h_{i j}=\frac{2 G m}{r}\left(1+\frac{L^{2}}{3 r^{2}}+\cdots\right) \delta_{i j},
\end{aligned}
$$

from which one may read off the Newtonian potential (5).

Moreover, all the metric components in (31) agree with those of (18) and not merely the $g_{00}$ component. In momentum space, this may be traced to the behaviour of $h_{\mu v}$ in the two pictures, namely (16) and (28). In (28) the factor of $-\frac{1}{3}$ in the non-leading term, as compared with factor $-\frac{1}{2}$ in the leading term, is attributable to the fact that the Kaluza-Klein gravitons are massive. Whereas in (16), it is because the CFT requires loop corrections with $\Pi_{2}(p)=-\frac{3}{2} \Pi_{1}(p)$, which is, in fact, satisfied, as far as the $\ln p^{2}$ term is concerned, since $a_{2}=-\frac{3}{2} a_{1}$.

We have thus demonstrated that the $1 / r^{3}$ corrections to Newton's law are identical between the Maldacena and Randall-Sundrum pictures. This was examined in the context of a single CFT corresponding to a one-sided brane-world scenario. Had we chosen instead to take the brane-world to be sitting between two patches $\mathrm{AdS}_{5}$ (one on either side), as was the case considered in [10,14], we would have obtained a factor of two in the relation between Newton's constants, with a corresponding factor in the propagator, equation (28). While this would ensure the correct four-dimensional behaviour of gravity, given in (31), the two-sided brane-world relation $G=G_{5} / L$ will modify the comparison with the one-loop CFT result, equation (24). To compensate for this mismatch, one may assume that the two-sided braneworld is dual to two copies of the CFT coupled to gravity, as is implicit in [10]. This leads to the natural picture that a one-sided brane corresponds to a single CFT while a two-sided brane corresponds to two CFTs.

An intriguing feature of this comparison of the gravitational potential in both pictures is a highlighting of the classical/quantum nature of this duality, as seen in the relation

$$
\Pi_{2}(p)+\mathcal{O}\left(G^{2}\right)=\frac{L}{4} \Delta_{K K}(p)
$$

The propagator for the continuum graviton modes in the Randall-Sundrum picture thus incorporates all quantum effects of matter on the brane. It may be worthwhile to examine this relation at the two-loop or higher level. Nevertheless, this agreement at one-loop lends strong support to the conjectured duality between the two pictures.

\section{Acknowledgments}

We would like to thank the organizers of the Feza Gursey Mememorial conference for their hospitality. The research was supported in part by DOE Grant DE-FG02-95ER40899

\section{References}

[1] Duff M J 1972 Problems in the classical and quantum theories of gravitation PhD Thesis Imperial College, London

[2] Duff M J 1974 Quantum corrections to the Schwarzschild solution Phys. Rev. D 91837 
[3] Capper D M, Duff M J and Halpern L 1974 Photon corrections to the graviton propagator Phys. Rev. D 10461

[4] Capper D M and Duff M J 1974 One-loop neutrino corrections to the graviton propagator Nucl. Phys. B 82147

[5] Capper D M 1975 On quantum corrections to the graviton propagator Nuovo Cimento A 2529

[6] Verlinde H 2000 Holography and compactification Nucl. Phys. B 580264 (Verlinde H 1999 Preprint hep-th/9906182)

[7] The complementarity and the Newton's law check were suggested independently by Witten E and Maldacena J in unpublished work

[8] Gubser S S 2001 AdS/CFT and gravity Phys. Rev. D 63084017

(Gubser S S 1999 Preprint hep-th/9912001)

[9] Giddings S B, Katz E and Randall L 2000 Linearized gravity in brane backgrounds J. High Energy Phys. JHEP03(2000)023

(Giddings S B, Katz E and Randall L 2000 Preprint hep-th/0002091)

[10] Hawking S W, Hertog T and Reall H S 2000 Brane new world Phys. Rev. D 62043501 (Hawking S W, Hertog T and Reall H S 2000 Preprint hep-th/0003052)

[11] Maldacena J 1998 The large $N$ limit of superconformal field theories and supergravity Adv. Theor. Math. Phys. 2231

(Maldacena J 1997 Preprint hep-th/9711200)

[12] Witten E 1998 Anti-de Sitter space and holography Adv. Theor. Math. Phys. 2253 (Witten E 1998 Preprint hep-th/9802150)

[13] Gubser S S, Klebanov I R and Polyakov A M 1998 Gauge theory correlators from non-critical string theory Phys. Lett. B 428105 (Gubser S S, Klebanov I R and Polyakov A M 1998 Preprint hep-th/9802109)

[14] Randall L and Sundrum R 1999 An alternative to compactification Phys. Rev. Lett. 834690 (Randall L and Sundrum R 1999 Preprint hep-th/9906064)

[15] Susskind L and Witten E 1998 The holographic bound in anti-de Sitter space Preprint hep-th/9805114

[16] Garriga J and Tanaka T 2000 Gravity in the Randall-Sundrum brane world Phys. Rev. Lett. 842778 (Garriga J and Tanaka T 1999 Preprint hep-th/9911055)

[17] Mück W, Viswanathan K S and Volovich I V 2000 Geodesics and Newton's law in brane backgrounds Phys. Rev. D 62165019

(Mück W, Viswanathan K S and Volovich I V 2000 Preprint hep-th/0002132)

[18] Capper D M and Duff M J 1974 Trace anomalies in dimensional regularization Nuovo Cimento A 23173

[19] Deser S, Duff M J and Isham C J 1976 Non-local conformal anomalies Nucl. Phys. B 1145

[20] Duff M J 1977 Observations on conformal anomalies Nucl. Phys. B 125334

[21] Duff M J 1994 Twenty years of the Weyl anomaly Class. Quantum Grav. 111387 (Duff M J 1993 Preprint hep-th/9308075)

[22] Henningson M and Skenderis K 1998 The holographic Weyl anomaly J. High Energy Phys. JHEP07(1998)023 (Henningson M and Skenderis K 1998 Preprint hep-th/9806087) 